\title{
Universality and Thouless energy in the supersymmetric Sachdev-Ye-Kitaev model
}

\author{
Antonio M. García-García, ${ }^{1, *}$ Yiyang Jia (贾抑扬), ${ }^{2, \dagger}$ and Jacobus J. M. Verbaarschot ${ }^{2, \$}$ \\ ${ }^{1}$ Shanghai Center for Complex Physics, School of Physics and Astronomy, \\ Shanghai Jiao Tong University, Shanghai 200240, China \\ ${ }^{2}$ Department of Physics and Astronomy, Stony Brook University, Stony Brook, New York 11794, USA
}

(Received 23 January 2018; published 4 May 2018)

\begin{abstract}
We investigate the supersymmetric Sachdev-Ye-Kitaev (SYK) model, $N$ Majorana fermions with infinite range interactions in $0+1$ dimensions. We have found that, close to the ground state $E \approx 0$, discrete symmetries alter qualitatively the spectral properties with respect to the non-supersymmetric SYK model. The average spectral density at finite $N$, which we compute analytically and numerically, grows exponentially with $N$ for $E \approx 0$. However the chiral condensate, which is normalized with respect the total number of eigenvalues, vanishes in the thermodynamic limit. Slightly above $E \approx 0$, the spectral density grows exponentially with the energy. Deep in the quantum regime, corresponding to the first $O(N)$ eigenvalues, the average spectral density is universal and well described by random matrix ensembles with chiral and superconducting discrete symmetries. The dynamics for $E \approx 0$ is investigated by level fluctuations. Also in this case we find excellent agreement with the prediction of chiral and superconducting random matrix ensembles for eigenvalue separations smaller than the Thouless energy, which seems to scale linearly with $N$. Deviations beyond the Thouless energy, which describes how ergodicity is approached, are universally characterized by a quadratic growth of the number variance. In the time domain, we have found analytically that the spectral form factor $g(t)$, obtained from the connected twolevel correlation function of the unfolded spectrum, decays as $1 / t^{2}$ for times shorter but comparable to the Thouless time with $g(0)$ related to the coefficient of the quadratic growth of the number variance. Our results provide further support that quantum black holes are ergodic and therefore can be classified by random matrix theory.
\end{abstract}

DOI: 10.1103/PhysRevD.97.106003

\section{INTRODUCTION}

Random matrix theory [1-6] is a powerful tool to explain universal features of complex quantum systems. In general, it is applicable for long timescales where the system has equilibrated and therefore its motion depends only on global symmetries, such as time reversal invariance and charge conjugation invariance. Quantitative agreement with random matrix theory indeed has been reported in a variety of problems: from disordered and quantum chaotic systems in the limit of negligible localization effects [7] to the spectrum of highly excited nuclei and lattice quantum chromodynamics (QCD) [8-10]. In the latter, it was found

\footnotetext{
*amgg@sjtu.edu.cn yiyang.jia@stonybrook.edu

; jacobus.verbaarschot@stonybrook.edu
}

Published by the American Physical Society under the terms of the Creative Commons Attribution 4.0 International license. Further distribution of this work must maintain attribution to the author(s) and the published article's title, journal citation, and DOI. Funded by SCOAP ${ }^{3}$. that both the density and level statistics of the low-lying eigenvalues of the QCD Dirac operator follow the predictions of random matrix theory where the choice of the ensemble, that labels the universality class, depends on the representation of the gauge group and the number and flavors. Despite its simplicity, random matrices can capture important dynamical features of realistic strongly correlated systems, for instance chiral symmetry breaking, a salient feature of nonperturbative QCD, related to the infrared limit of the spectral density [11] of the QCD Dirac operator.

Despite its success, the random matrix approach has obvious limitations as for sufficiently short times where the quantum dynamics is not universal. In certain cases, such as noninteracting disordered metals and quantum chaotic systems, where there is a good understanding of these corrections, it is possible to estimate the energy or time scale, usually called Thouless energy or time, where nonuniversal corrections are expected and to even get quantitative information on the dynamics at this intermediate scale by studying the corrections to the random matrix results [12]. For strongly interacting systems, the situation 
is less clear though several approaches have been developed to tackle this problem in the context of condensed matter [13], QCD [14] and nuclear physics [15-19].

In the latter, fermionic models with infinity range twobody interactions were introduced as a natural generalization of random matrix models with the expectation of a larger range of applicability while keeping some analytical control. More recently, research on this problem has received an important new impetus after the realization [20] that $N$ fermions with infinite range two-body interactions, now called Sachdev-Ye-Kitaev (SYK) model [20-22], could be a toy model for holography, namely, the infrared limit, which is described by a Schwarzian action could be dual to a certain quantum black hole in an anti-de Sitter space in two bulk dimensions.

The SYK model in the strong coupling limit has a maximal Lyapunov exponent [20,23-27], a finite zero temperature entropy $[20,21,28,29]$, a linear specific heat in the low temperature limit [30,31], the density of the low energy excitations grows exponentially [32-35] and short range spectral correlations are given by random matrix theory $[33,36,37]$. We note that the observation of a nonzero Lyapunov exponent at the Ehrenfest time and random matrix like level statistics at the Heisenberg time, a much larger time scale of the order of mean level spacing, are both signatures of quantum chaos. Based on thermodynamic properties, a similar pattern of conformal symmetry breaking and the existence of quantum chaos characterized by a Lyapunov exponent that saturates a bound on chaos [23], it has been conjectured $[38,39]$ that the gravity-dual of the SYK model is the Jackiw-Teitelboim background [40,41], a near $\mathrm{AdS}_{2}$ dilatonic geometry that is also described by a Schwarzian action in the low temperature limit.

Recently, different extensions of the SYK model have been studied including several fermionic flavors [42,43], higher dimensions [44-48], one plus two-body interactions [49-51], with supersymmetry [52-59] and with no disorder $[37,60,61]$.

In these supersymmetric SYK models, global symmetries, that depend on the number of Majoranas, agree $[55,56]$ with those of the chiral and superconducting [62] ensembles of random matrices [10]. Its gravity dual has been identified [63] as a supersymmetric extension of the Jackiw-Teitelboim background mentioned above based on the fact that both systems are described by a Schwarzian action in the close-to-extremal limit. Since global symmetries of the non-supersymmetric model match those of the standard Wigner-Dyson ensembles, the random matrix correspondence of the supersymmetric SYK model extends this to most of the known [62] universality classes in random matrix theory.

This raises several questions: do SYK models with different global symmetries still keep most of the features expected in a theory with gravity dual such as quantum chaos, exponential increase of low energy excitations or a finite entropy at zero temperature? What is the precise window of universality in which random matrix theory is applicable and how are corrections beyond this universal regime?

In this paper we aim to answer these questions. We study the spectral density, thermodynamic properties and level statistics in a supersymmetric SYK model by analytical and exact diagonalization techniques. Our main results are that this chiral SYK model still has all the expected features of a gravity dual: finite entropy at zero temperature, exponential increase of low energy excitations and excellent agreement of level statistics with random matrix theory predictions for sufficiently long time or small energy separations. We also show that not only level statistics but also the spectral density in the infrared limit is universal and is given by random matrix theory. We study quantitatively deviations from these universal results, observed for energies (times) larger (smaller) than the so called Thouless energy (time) [12]. The number variance grows quadratically for eigenvalue separations larger than the Thouless energy and the spectral form factor decays as a power law for times shorter than the Thouless time.

The paper is organized as follows. In Sec. II, we start with the definition of the model and then discuss analytical results for the spectral density. We also discuss the spectral resolvent of the supercharge and the thermodynamic properties of the Hamiltonian. In Sec. III we compare numerical results for the microscopic spectral density with random matrix predictions. Level statistics and its comparison with random matrix theory, including deviations for (small) large (time) energies, are discussed in Sec. IV. Concluding remarks are made in Sec. V. In Appendix A we work out the large $N$ limit of the Q-Hermite result for the spectral density while a simple analytical form for the resolvent of the SYK model is obtained in Appendix B.

\section{MODEL, AVERAGE SPECTRAL DENSITY AND THERMODYNAMIC PROPERTIES}

\section{A. The SYK model}

We investigate a SYK model, namely $N$ Majorana fermions with infinite range $q$-body interactions, for $q=3$. The Hamiltonian is given by the square of the supercharge $Q$ [52]

$$
H=Q^{2},
$$

where the supercharge is defined by

$$
Q=i \sum_{i, j, k=1}^{N} J_{i j k} \gamma_{i} \gamma_{j} \gamma_{k}
$$

with $\gamma_{i}$ Majorana fermions defined by the following algebraic relation:

$$
\left\{\gamma_{i}, \gamma_{j}\right\}=\delta_{i j}
$$


which is also satisfied by Euclidean Dirac $\gamma$ matrices. The coupling $J_{i j k}$ is chosen to be a Gaussian random variable with probability distribution

$$
P\left(J_{i j k}\right)=\sqrt{\frac{N^{2}}{36 \pi J^{2}}} \exp \left(-\frac{N^{2} J_{i j k}^{2}}{36 J^{2}}\right),
$$

where $J$ sets the scale of the distribution. Unless specified otherwise $J \equiv 1$.

This Hamiltonian is supersymmetric with global symmetries that, depending on $N$, correspond to one of the chiral and superconducting random matrix ensembles $[52,56]$. In this paper we will study the thermodynamical properties of the Hamiltonian, but we will analyze the spectral properties of the supercharge for $E \sim 0$ corresponding to the ground state and low energy excitations of the Hamiltonian.

\section{B. The spectral density of the supercharge}

The average spectral density for even $q$, computed in Ref. [32] by an explicit evaluation of the moments, turned out to be given by the weight function of the $Q$-Hermite polynomials with $Q=\eta$ where $\eta(N, q)$ is a suppression factor related to the commutation of two products of $q$ Majorana operators. A similar calculation can be carried out for odd $q$. The average density is still given by the same analytical expression but with a negative suppression parameter (for even $q, \eta$ can also become negative for small values of $N$ ):

$$
\eta=\left(\begin{array}{c}
N \\
q
\end{array}\right)^{-1} \sum_{r=0}^{q}(-1)^{r+q}\left(\begin{array}{c}
q \\
r
\end{array}\right)\left(\begin{array}{c}
N-q \\
q-r
\end{array}\right)
$$

In terms of $|\eta|$ the average density can be rewritten as

$$
\begin{aligned}
& \rho(E)=c_{N} \sqrt{1-\frac{E^{2}}{E_{0}^{2}}} \prod_{k=1}^{\infty}\left[1-4 \frac{E^{2}}{E_{0}^{2}} \frac{1}{2+|\eta|^{2 k}+|\eta|^{-2 k}}\right] \\
& \times\left[1-4 \frac{E^{2}}{E_{0}^{2}} \frac{1}{2-|\eta|^{2 k-1}-|\eta|^{-2 k+1}}\right] \text {. }
\end{aligned}
$$

Note that this expression is valid for both even and odd $q$. After a Poisson resummation, an explicit evaluation of the resulting integrals is possible (see Appendix A). In the large $N$ limit, it simplifies to,

$$
\begin{aligned}
\rho_{\text {asym }}(E)= & c_{N} \cosh \left(\frac{\pi \arcsin \left(E / E_{0}\right)}{\log |\eta|}\right) \\
& \times \exp \left[2 \frac{\arcsin ^{2}\left(E / E_{0}\right)}{\log |\eta|}\right]
\end{aligned}
$$

provided that the absolute value of the energy is away from $E_{0}$ given by

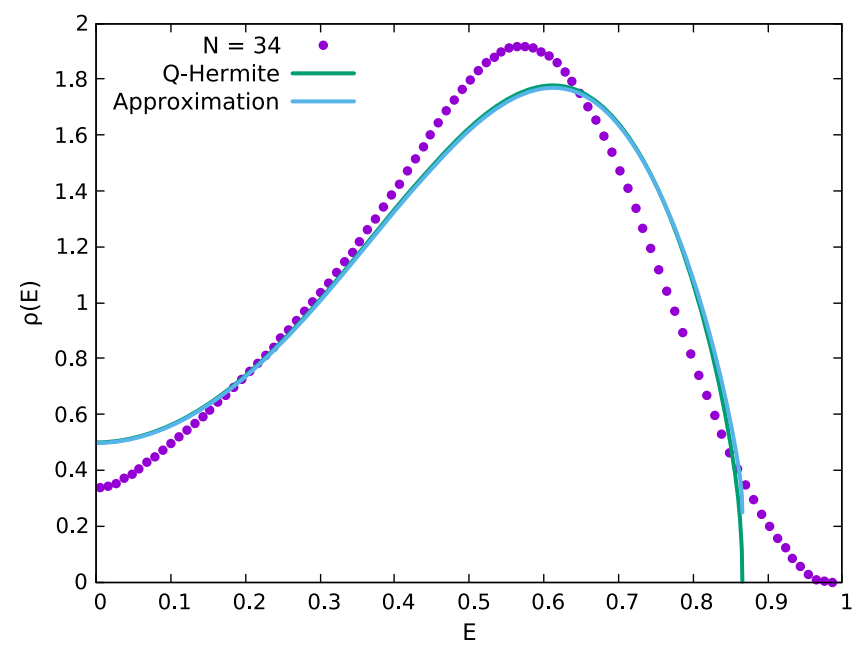

FIG. 1. Average spectral density of the supercharge $Q$ (with Hamiltonian $H=Q^{2}$ ) of the SYK model Eq. (2) for $q=3$ and $N=34$. We find excellent agreement between the analytical Q-Hermite result Eq. (6) and the approximation Eq. (7). However, agreement with the exact diagonalization results (solid dots), employing $5 \times 10^{6}$ eigenvalues, is only qualitative.

$$
E_{0}=\frac{4 \sigma^{2}}{1-\eta}
$$

where

$$
\sigma^{2}=\left(\begin{array}{l}
N \\
q
\end{array}\right) \sigma_{0}^{2}
$$

with $\sigma_{0}$ the standard deviation of $J$. For even $q$, this asymptotic expression turned out to be not only an excellent approximation of the Q-Hermite result Eq. (6) but also of the exact density obtained by numerical diagonalization of Eq. (2). Results depicted in Fig. 1 confirm that Eq. (7) is still an excellent approximation of the $Q$-Hermite results. However, the agreement with the spectral density from exact diagonalization for $N=34$ is only qualitative. Since correction terms to the moments in the Q-Hermite approximation Eq. (6) are of order $q / N$ the excellent agreement for even $q$ was unexpected.

\section{The resolvent}

In this section, we study the resolvent of the supercharge of the SYK model which is an alternative way to investigate its average spectral properties. It is defined by

$$
G(z)=\sum_{\lambda_{k}} \frac{1}{z+\lambda_{k}},
$$

where the $\lambda_{k}$ are the eigenvalues of $Q$. The spectral density is given by the discontinuity of the resolvent across the real axis. Conversely, the resolvent Eq. (10) follows by integrating over the spectral density. Because the spectrum of $Q$ in Eq. (2) is symmetric under $\lambda_{k} \rightarrow-\lambda_{k}$, the resolvent on the imaginary axis 


$$
i G(i s)=\frac{1}{\mathcal{N}} \sum_{\lambda_{k}>0} \frac{2 s}{\lambda_{k}^{2}+s^{2}}
$$

is purely imaginary (so that $i G(i s)$ is real). According to the Banks-Casher relation [11], the resolvent in the $s \rightarrow 0$ and the thermodynamic limit is directly related to the spectral density at $E=0$. In the context of QCD, a finite value in this limit signals the spontaneous breaking of chiral symmetry, one of its most relevant low energy features. In the supersymmetric SYK model, it is unclear whether this interpretation is exactly applicable. However, it is still of interest to study it as a finite spectral density at the origin is a strong indication of a highly entangled ground state.

Moreover, we have derived a compact analytical expression based on the known $[64,65]$ spectral density for Q-Hermite polynomials. We leave the details of the calculation to the Appendix B and state here the final result [64]:

$i G(i s)=\frac{\sqrt{1-\eta}}{\sigma} \sum_{k=0}^{\infty} \frac{\eta^{k(k+1) / 2}}{\left(\frac{s \sqrt{1-\eta}}{2 \sigma}+\sqrt{\frac{s^{2}(1-\eta)}{4 \sigma^{2}}+1}\right)^{2 k+1}}$.

In Fig. 2 we depict results for the resolvent as a function of $s$ for different values of $N$. We find excellent agreement with the analytical prediction Eq. (12) except for small $s$, dominated by high moments, where we expect the Q-Hermite result to be less accurate. In order to explore numerically the limit $s \rightarrow 0, N \rightarrow \infty$ we carry out a finite size scaling analysis in $N$ for a small $s \sim 0.01$. We fit the $N$

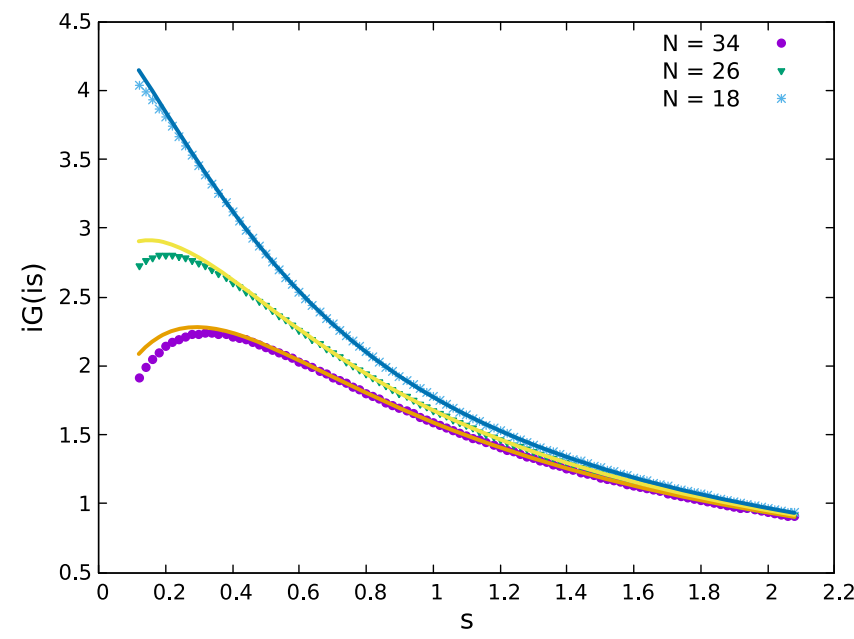

FIG. 2. Resolvent $i G(i s)$ Eq. (11) for the SYK model for $q=3$ as a function of the parameter $s$ for different values of $N$ compared with the analytical result Eq. (12). After a finite size scaling analysis, we find that the $N$-dependence of $\log G(0)$ is well fitted by $\log G(0) \approx-0.0695 N$ in agreement with the value of the $q$-dependent part of the zero temperature entropy (15). We find excellent agreement with the analytical expression Eq. (12) based on the Q-Hermite density except for very small $s$. dependence of the logarithm of the resolvent by a function $a+b N$ where $a$ and $b$ are fitting parameters. The slope is equal to the nontrivial part of the entropy density. We find $b \approx-0.0695$ which agrees with the analytical result $-\pi^{2} /\left(16 q^{2}\right)$ to be discussed in the next subsection.

\section{Thermodynamic properties}

In this subsection we study the thermodynamic properties of the model in the large $N$ and low temperature limit where the asymptotic average density Eq. (7) is expected to be a good approximation. We note that because the Hamiltonian $H=Q^{2}$, where $Q$ is the supercharge, the low temperature limit is controlled by states with positive energy close to $E=0$. In contrast with even $q$ where for large $N, E_{0} \sim N$, for odd $q$ when $\eta<0, E_{0} \sim \sqrt{N}$. The region close to the origin for odd $q$ case is therefore qualitatively different from the even $q$ case where the ground state energy scales with $N$ as should be the case for a fermionic system.

The entropy at zero temperature $s_{0}$ follows from the prefactor $c_{N}$ in Eq. (6). Although this constant is known analytically, see Appendix A, its large $N$ limit can simply be determined from the condition

$$
\int d E \rho_{\text {asym }}(E) d E=2^{N / 2} .
$$

Evaluating the integral by a saddle point approximation and using that in this limit $\log |\eta| \sim-2 q^{2} / N$ we find

$$
c_{N}=e^{N / 2 \log 2-N \pi^{2} / 16 q^{2}} .
$$

Since the Q-Hermite approximation only has a nontrivial large $N$ limit when $q^{2} / N$ is kept fixed, it can only yield a $1 / q^{2}$ correction to the zero temperature entropy which is given by

$$
s_{0}=\log c_{N}=N\left(\frac{1}{2} \log 2-\frac{\pi^{2}}{16 q^{2}}\right) .
$$

This result agrees up to order $1 / q^{2}$ with the exact result [52] of $s_{0}$ obtained by using path integral techniques. We note that $s_{0}$ is larger than the value of $s_{0}$ in the even $q$ case, where the nontrivial contribution to the entropy is $\pi^{2} / 4 q^{2}$. This larger entropy, which suggests a highly entangled ground state, is likely a consequence of the special properties at $E \approx 0$ due to the chiral symmetry of the model.

As a further confirmation of this, we calculate the low temperature limit of the free energy from the asymptotic spectral density Eq. (7). We show that it agrees with the large $q$ limit of the free energy for odd $q$ obtained in [52].

Since the Hamiltonian $H=Q^{2}$ and $\rho_{\text {asym }}(E)$ is the eigenvalue density of $Q$, the partition function is given by

$$
Z(\beta)=\int d E \rho_{\text {asym }}(E) e^{-\beta E^{2}} .
$$


For large $N$, the integral can be evaluated by a saddle point approximation with saddle point equation given by

$$
\begin{aligned}
& -\frac{\pi}{E_{0} \log |\eta|} \frac{1}{\sqrt{1-\left(E / E_{0}\right)^{2}}} \\
& +\frac{4}{E_{0} \log |\eta|} \frac{\arcsin \left(E / E_{0}\right)}{\sqrt{1-\left(E / E_{0}\right)^{2}}}-2 \beta E=0 .
\end{aligned}
$$

The free energy is therefore given by

$$
\begin{aligned}
-\beta F= & \frac{N}{2} \log 2+\frac{\pi^{2}}{8 \log |\eta|}-\frac{\pi \arcsin \left(E / E_{0}\right)}{\log |\eta|} \\
& +2 \frac{\arcsin ^{2}\left(E / E_{0}\right)}{\log |\eta|}-\beta E^{2} .
\end{aligned}
$$

Substituting

$$
E=E_{0} \sin ((\pi-v) / 4)
$$

and using the saddle point equation

$$
\frac{-v}{E_{0}^{2} \log |\eta| \cos (v / 2)}-\beta=0,
$$

the free energy can be rewritten as

$-\beta F=\frac{N}{2} \log 2+\frac{v^{2}}{8 \log |\eta|}+\frac{v}{2 \log |\eta|} \tan ((\pi-v) / 4)$.

If we identify $-E_{0}^{2} \log |\eta|=\mathcal{J}$ this is exactly the saddle point equation obtained in [52]. Taking the large $N$ limit, it gives the free energy obtained in [52],

$-\beta F=\frac{N}{2} \log 2-N \frac{v^{2}}{16 q^{2}}-N \frac{\beta \mathcal{J}}{4 q^{2}}+N \frac{v}{2 q^{2}} \tan ((\pi-v) / 4)$

with the constant energy $N \mathcal{J} / 4 q^{2}$ subtracted. In the lowtemperature limit, the solution of the saddle point equation is given by $v=\pi$ resulting in the free energy

$$
-\beta F=\frac{N}{2} \log 2-N \frac{\pi^{2}}{16 q^{2}}-N \frac{\beta \mathcal{J}}{4 q^{2}}+O(1 / \beta) .
$$

The conclusion of this analysis is that the Q-Hermite form of the spectral density which at large $N$ only has a nontrivial limit when $q^{2} / N$ is kept fixed reproduces the leading $1 / q^{2}$ correction to the free energy. Contrary to the even $q$ case, for odd $q$ the low-temperature limit is determined by energies close to zero. In the following section, we focus on this region only.

\section{UNIVERSAL MICROSCOPIC SPECTRAL DENSITY}

Depending on $N$, the supersymmetric SYK model [52,55] has additional chiral and discrete symmetries. The analysis of thermodynamic properties has revealed that the main differences with respect to the original SYK model are in the region close to the ground state $E \approx 0$. It is well known [9] that, precisely in this region, the microscopic spectral density $\rho_{M}(E)$ defined by

$$
\rho_{M}(E)=\Delta \rho\left(\frac{E}{\Delta}\right)
$$

with $\Delta$ the level spacing near $E=0$, is universal and given by random matrix theory.

Explicit analytical expressions are known for all universality classes $[9,66,67]$. This is in stark contrast with Wigner-Dyson ensemble where only the spectral correlations, not the density, at the scale of the mean level spacing have universal features. The origin of this universality lies in the additional rigidity of the spectrum at $E \approx 0$ imposed by chiral symmetry which requires the nonzero eigenvalues to occur in pairs $\pm \lambda_{k}$. In realistic systems, like $\mathrm{QCD}$, this is only observed deep in the quantum regime corresponding to the first $O\left(F_{\pi}^{2} \sqrt{V}\right)$ eigenvalues with $F_{\pi}$ the pion decay constant and $V$ the space-time volume. For practical lattice QCD simulations this amounts to only the first few eigenvalues above $E=0$. Since the supersymmetric SYK model has the same global symmetries as those of the mentioned random matrix ensembles, it is natural to inquire whether the spectral density is also universal.

For that purpose, we compute the spectral density by exact diagonalization techniques. For any given $N$, we calculate at least $8 \times 10^{7}$ eigenvalues. Specifically, for $N=26,30,32,34$, the number of disorder realizations is $27340,4430,1300$ and 540, respectively. Such a large number of eigenvalues is necessary to suppress statistical fluctuations. The SYK microscopic spectral density for the first few eigenvalues, depicted in Fig. 3, shows an excellent agreement with random matrix ensemble belonging to the same universality class. We note that this is a completely parameter-free comparison.

For the chiral symplectic ensemble, we observe a suppression of the oscillations for eigenvalue sufficiently far from the origin. This is consistent with results from other systems such as QCD [9] where dynamical features not present in the random matrix ensemble tend to soften the effect of discrete symmetries. The scale at which the oscillations disappear, known as the Thouless energy, increases with $N$, and although an estimate of the precise $N$ dependence is hard, it seems that it scales linearly with $N$. We note the distribution of the first eigenvalue was already worked out in Ref. [56] where agreement with random matrix theory was also found. 

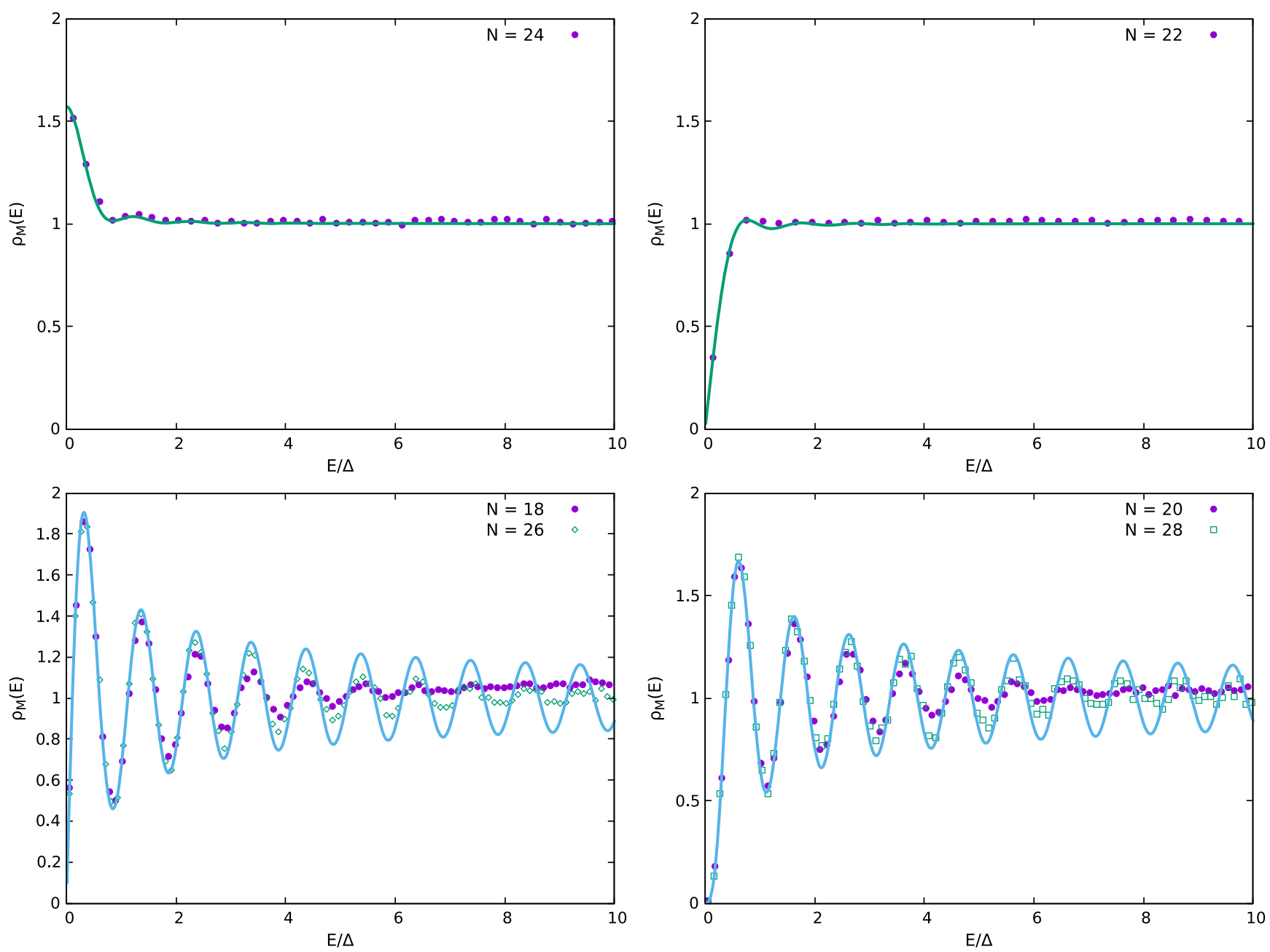

FIG. 3. Microscopic spectral density $\rho_{M}$ near $E=0$ in units of the mean level spacing $\Delta$. We compare $\rho_{M}$ for the supercharge of the SYK model with $q=3$, see Eq. (2), with the predictions of random matrix theory (solid curves). As was shown in [55], SYK models with different values of $N$ have different discrete global symmetries and therefore must be compared to random matrix ensembles belonging to the corresponding universality classes. For instance, $N=18$ and $N=26$ belongs to the $D I I I$ ensemble, $N=20$ and $N=28$ to the chiral symplectic ensemble (chGSE), $N=24$ to the chiral orthogonal ensemble (chGOE), and $N=22$ to the $C I$ ensemble. Interestingly, we observe an excellent agreement with the random matrix prediction for the first few low lying eigenvalues. The oscillations for the microscopic spectral density of the SYK model in the cases of DIII and chGSE (bottom row), are eventually washed out as the effect of discrete symmetries is strong only very close to $E=0$. Analytical expressions for all random matrix ensembles can be found in Refs. $[9,66,67]$.

\section{UNIVERSALITY IN LEVEL STATISTICS AND QUANTITATIVE ESTIMATION OF THE THOULESS ENERGY}

We now proceed to the study of dynamical properties of the SYK model by studying level statistics beyond the Thouless energy. In the nonsupersymmetric SYK model, it was found [32,33] that in both the bulk of the spectrum and the region close to the ground state, spectral correlations for small eigenvalue separations are well described by random matrix theory. This is an indication that for sufficiently long times, of the order of the Heisenberg time, the SYK model is still quantum chaotic and has reached an ergodic state where the dynamics is universal since it depends only on the global symmetry of the system.

We focus again in the low energy region $E \approx 0$ where we expect more differences with respect to the SYK model with even $q$. Since agreement with random matrix theory is expected for short-range spectral correlations, we investigate the number variance, a popular choice to characterize long range spectral correlations which will allow us a more systematic description of the corrections to the random matrix results. It is defined as the variance of the number of levels $\tilde{N}(\epsilon)$ in an energy interval of width $\epsilon$ (in units of the mean level spacing):

$$
\Sigma^{2}(\epsilon)=\left\langle\tilde{N}^{2}(\epsilon)\right\rangle-\langle\tilde{N}(\epsilon)\rangle^{2} .
$$



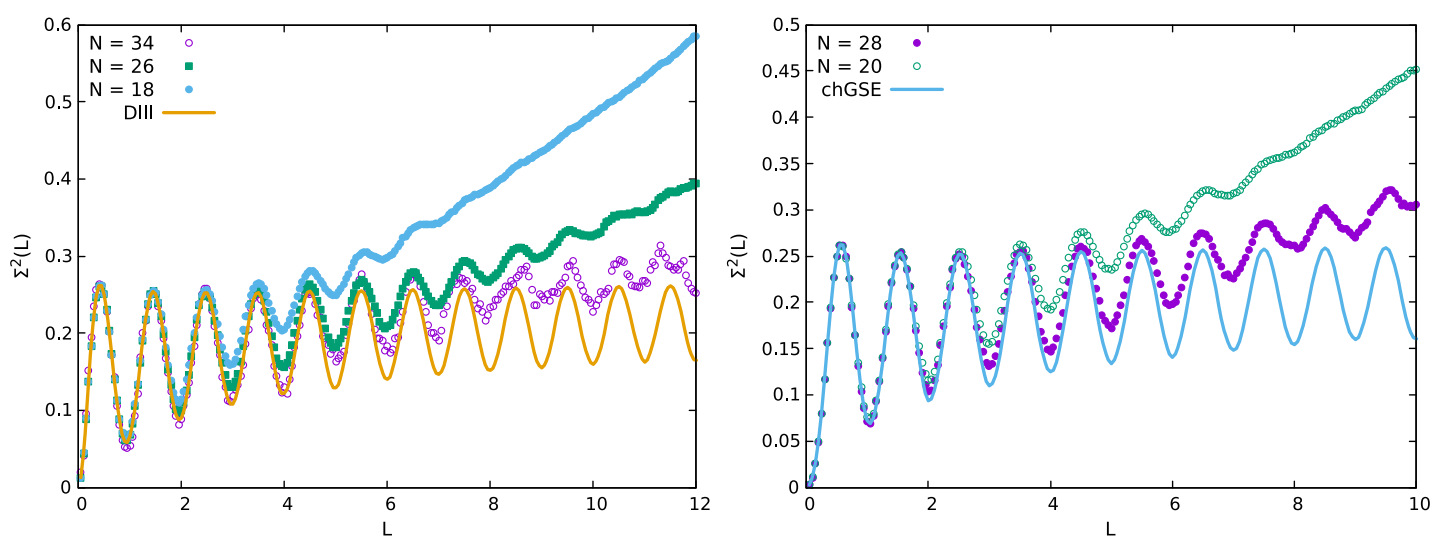

FIG. 4. Number variance $\Sigma^{2}(L)$ Eq. (25) for small $L$ and different $N$ 's. Excellent agreement with random matrix theory is observed for sufficiently small $L$. This is an indication that SYK for odd $q$ is also quantum chaotic for sufficiently long times (small energies). The point after which deviations are observed, especially its scaling with $N$, provides useful information on the quantum dynamics prior to complete relaxation. Our numerical results are consistent with a point of departure that roughly scales with $N$.

In order to proceed, we have to unfold the spectrum, namely, to rescale it so that the mean level spacing is energy independent. In addition we use the connected two-point function to study spectral correlations. The removal of the one-point function contribution is crucial to relate results from level statistics to dynamical features of the system.

We employ two unfolding methods: a polynomial of fifth degree fitting for the whole averaged spectrum and the splines method where consecutive groups of several eigenvalues, at least of the order of the spectral window to be investigated, are fitted to a linear or quadratic polynomial. Results from both methods are similar so we stick to the latter which provides a slightly more accurate fitting for the eigenvalues closest to the origin. In terms of the unfolded spectrum $\langle\tilde{N}(\epsilon)\rangle=\epsilon$, so $\langle\tilde{N}\rangle \equiv L$ where $L$ is the number of unfolded eigenvalues in the window of interest. The number variance can be expressed as the double integral of the connected two-level correlation function whose analytical expression is known for all ensembles of random matrices [66]. However, it is often simpler to generate these curves from numerical exact diagonalization of random matrices, which we will do for comparison to the SYK model. The number variance for $L<10$, starting from $E=0$, is shown in Fig. 4 for different values of $N$, corresponding to different universality classes. In all cases we find excellent agreement with the random matrix prediction for the first few eigenvalues. As was expected, because the SYK model is quantum chaotic, the agreement extends to more eigenvalues as $N$ increases. The point of departure from the random matrix prediction, usually called Thouless energy, seems to scale as $N^{\alpha}$ with $\alpha \sim 1$ for all universality classes. This is consistent with previous results for the microscopic spectral density. However the value of $\alpha$, and therefore of the Thouless energy, for the nonsupersymmetric SYK models $(q>2$ even) seems to be
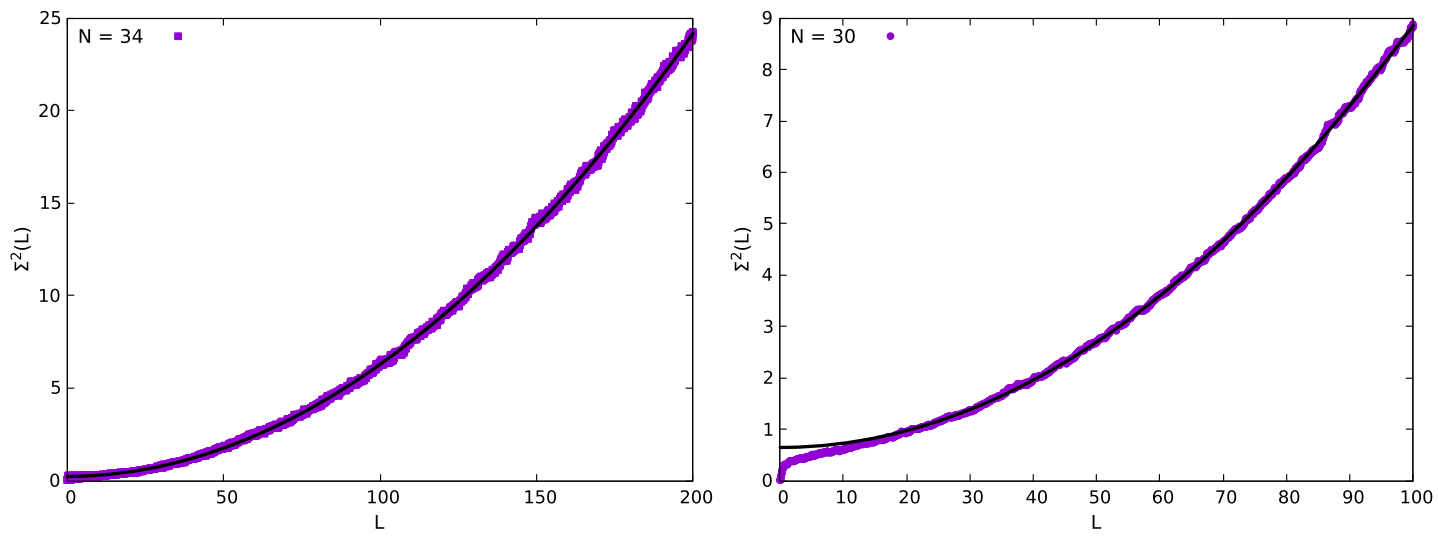

FIG. 5. Number variance $\Sigma^{2}(L)$ Eq. (25) for $N=30$ and $N=34$. Deviations from the logarithmic growth predicted by random matrix theory are clearly observed. We have fitted the growth (solid curves) to a function $\sim L^{\alpha}$. For $N$ sufficiently large $\alpha \approx 2$ ( $\alpha \approx 1.99$ for $N=34$ and $\alpha \approx 1.98$ for $N=30$ ). For sufficiently small $N$, we find a bit slower growth but we believe that this is a finite size effect of no physical relevance. 

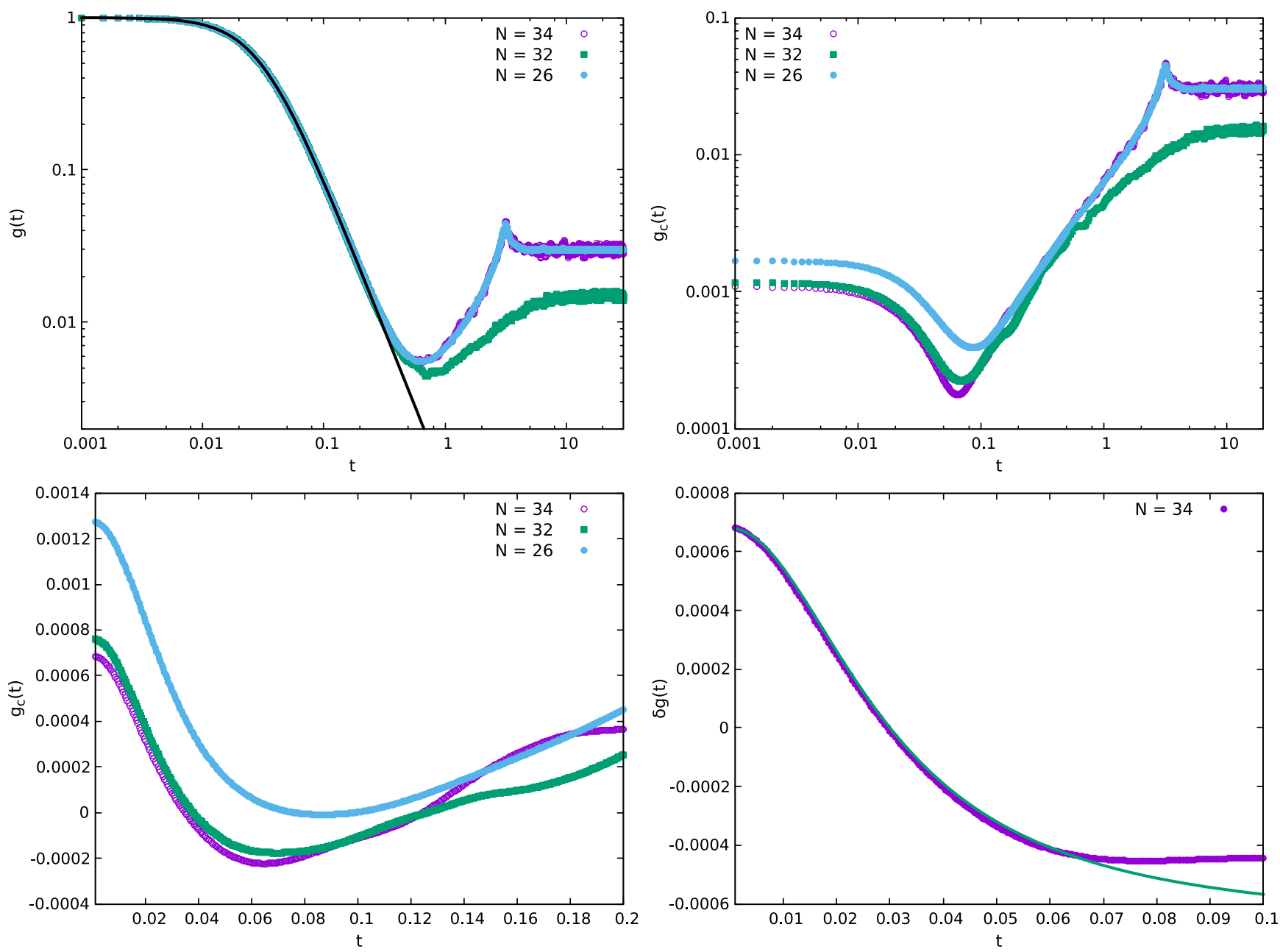

FIG. 6. Top-Left: Spectral form factor $g(t)$ Eq. (29), with $t$ in units of $J$ and $\hbar \equiv 1$, for $\beta=0.03$ obtained from the unfolded spectrum of the supercharge Eq. (2) and different values of $N$. The solid black curve represents the disconnected part of $g(t)$, $g_{d}(t)=\beta^{2} /\left(\beta^{2}+t^{2}\right)$, the leading contribution to $g(t)$ for short times, that must be subtracted from $g(t)$ in order to extract the Thouless time $t_{T}$. Top-Right: Connected spectral form factor $g_{c}=g-g_{d}$. For sufficiently long times, we observe a dip (correlation hole) at $t_{T}$ followed by a ramp and eventual saturation, all typical features of quantum chaotic system and also predicted by random matrix theory. The peak for $N=32$ and $N=34$ is a known feature of quantum chaotic systems with symplectic symmetry. We have slightly shifted all curves by 0.0004 so that we can plot them in a log-scale. Bottom-left: Zoom in of $g_{c}$ for short times. The position of the correlation hole seems to roughly scale with $N$. Bottom-right: the deviation from the random matrix theory result $\delta g(t)$ for $t<t_{T}$, before the dip is formed. Assuming fully chaotic eigenfunctions, $\delta g(t)$ describes how the SYK model approaches equilibrium. We have found that the numerical result agrees well with the theoretical prediction Eq. (32) (solid curve) which decays as $t^{-2}$, as $t_{T}$ is approached.

much larger $(\alpha \approx 2)$ [36]. For even $q$, there is an analytical argument that indeed $\alpha=2$ [68]. A reason for that behavior is that, for smaller $q$, the Hamiltonian is sparser and therefore it takes more time, so less energy, to explore the full phase space available.

Beyond the Thouless energy, we observe a growth of the number variance much faster than the logarithmic growth predicted by random matrix theory. Our results, shown in Fig. 5 for different values of $N$, are fully consistent with a quadratic growth $\sim L^{2}$ which is in agreement with the result obtained in Ref. [68]. This quadratic growth can be understood as follows: in a nonlinear sigma model massive modes contribute a constant $c_{M}$ to the connected two-level correlation function,

$$
\rho_{2, c}\left(\lambda_{1}, \lambda_{2}\right)=\rho_{2, c}^{\mathrm{RMT}}\left(\lambda_{1}, \lambda_{2}\right)+c_{M}-\delta \rho_{2}\left(\lambda_{1}, \lambda_{2}\right),
$$

where the (short-range) correction $\delta \rho_{2}\left(\lambda_{1}, \lambda_{2}\right)$ is chosen such that the exact sum rule is satisfied

$$
\int d \lambda_{1} \rho_{2, c}\left(\lambda_{1}, \lambda_{2}\right)=0
$$

Note that $\rho_{2, c}^{\mathrm{RMT}}$ satisfies this sum rule. The constant term in the two-point function gives a quadratic contribution to the number variance. From Fig. 5 we find that the constant term in Eq. (26) is well approximated by

$$
c_{M} \approx \frac{\pi}{4} \frac{1}{N^{2}} .
$$


The power law increase of the number variance is reminiscent of the result for weakly disordered metals $\sim L^{d / 2}$ [12], where $d$ is the spatial dimensionality of the system though it is not clear to us whether it is possible to push this analogy any further.

\section{A. Spectral form factor}

In a time representation, the Thouless energy, and the subsequent quadratic growth of the number variance, are interpreted as the minimum time after which the dynamics is universal and how this universal regime is approached respectively. In order to explore these time scales, we compute the spectral form factor [69-72] with the unfolded spectrum,

$$
g(t)=\frac{\left\langle Z^{*}(t) Z(0)\right\rangle}{\langle Z(0)\rangle^{2}}
$$

where $Z=\sum_{i} e^{i \lambda_{i} t-\beta \lambda_{i}}$ with $\lambda_{i}$ the unfolded eigenvalues and $\beta$ the inverse temperature. We also remove the disconnected part $g_{d}(t)=\beta^{2} /\left(\beta^{2}+t^{2}\right)$ related to the one-point function. Although not always employed in the literature [33], it is necessary to work with unfolded eigenvalues and connected two-point functions for a correct determination of the Thouless energy.

We illustrate this point in Fig. 6 where we compute the full spectral form factor, for $\beta=0.03$, together with the connected part $g_{c}=g-g_{d}$. We observe the ramp and saturation expected from random matrix theory in both cases. We also observe a dip (correlation hole) for short times. Only for $g_{c}$ this is related to the Thouless time, a time scale that describes the limit of applicability of random matrix theory. The dip (correlation hole) occurs for $N=34$ at $t \sim 0.1$, about $1 / 10$ of the Heisenberg time. This is roughly consistent with the estimation of the Thouless energy from the number variance in Fig. 4 which was computed by removing the double degeneracy (for $g(t)$ we kept the double degeneracy) and also using the unfolded spectrum and the connected part of the two-level correlation function.

The scaling of $t_{T}$, see Fig. 6 (bottom left), seems to be linear with $N$, also consistent with the number variance estimation. For $t<t_{T}$, the spectral form factor deviates from the random matrix theory prediction. Simple perturbation theory [70,71], valid for $t \ll t_{T}$, shows that

$$
\delta g(t) \equiv g_{c}(t)-g_{c}^{\mathrm{RMT}}(t) \sim \beta^{2}-t^{2} .
$$

Similarly, for $t \lesssim t_{T}$, the constant contribution $c_{M}$ in Eq. (26) controls $\delta g(t)$ so

$$
\delta g\left(t \lesssim t_{T}\right) \approx g(0) \frac{\beta^{2}}{\beta^{2}+t^{2}} .
$$

with $g(0) \approx c_{M}$. More specifically, for $N=30$ and $N=34$ the coefficients of the quadratic dependence of the number variance $c_{M}$ are 0.00080 and 0.00062 , respectively, while $g(0)$ are equal to 0.00075 and 0.00068 .

In Fig. 6 (bottom right) we compare the numerical $\delta g(t)$ with a simple interpolating expression

$$
\delta g(t)=g(0) \frac{\beta^{2}-t^{2}}{\beta^{2}+t^{2}},
$$

valid for any $t<t_{T}$. The agreement is excellent until times very close to $t_{T}$.

As for the number variance, our results are reminiscent to those for noninteracting weakly disordered metals in more than two spatial dimensions where a power-law decay was observed $[12,73]$. However there are important differences. In our case, diffusion is in Fock space and it is unclear whether the techniques and ideas employed for a noninteracting problem can be translated to the interacting SYK model.

\section{OUTLOOK, HOLOGRAPHIC INTERPRETATION AND CONCLUSIONS}

We have studied the spectral and thermodynamic properties of the supersymmetric SYK model (odd $q$ ). We have found that the microscopic spectral density corresponding to the $O(N)$ smallest eigenvalues is universal, and is well described by chiral or superconducting random matrix ensembles depending on the value of $N$. The average spectral density, computed analytically and numerically, for $E \approx 0$ grows exponentially with $N$. However the chiral condensate, obtained from the spectral resolvent, which is normalized with respect to the total number of eigenvalues, vanishes in the thermodynamic limit.

For slightly larger energies, the average spectral density grows exponentially. Spectral correlations in the region $E \approx 0$ also agree with the random matrix prediction for sufficiently small eigenvalue separations. For eigenvalue separation larger than the Thouless energy, which roughly scales with $N$, we observe deviations from random matrix theory which are characterized by a quadratic growth of the number variance. We have also found a simple analytical expression for the spectral form factor, valid for times shorter than the Thouless time, that provides useful information about how the SYK model approaches ergodicity.

The results of this paper raise some further questions. For instance, the observation of level statistics described by random matrix theory for long times in the supersymmetric SYK model strongly suggests that its gravity dual [63] should also share this feature. It would be very interesting to carry out a direct demonstration of this fact as it would be an indication this is a universal feature of quantum black holes in gravity theories with a field theory dual. It would also be of interest to understand why the entropy at zero temperature is larger in the supersymmetric case with respect to the nonsupersymmetric one. 


\section{ACKNOWLEDGMENTS}

Aurelio Bermúdez (A. M. G.), Bruno Loureiro (A. M. G.), Alex Kamenev (J. V.) and Lea Santos (J. V. and A. M.G) are thanked for illuminating discussions. Y. J. and J. V. acknowledge partial support from U.S. DOE Grant No. DE-FAG-88FR40388.

\section{APPENDIX A: SPECTRAL DENSITY FOR ODD $q$}

In this Appendix we calculate the large $N$ limit of the spectral density for odd $q$.

For negative $\eta$ the spectral density can be written as

$$
\begin{aligned}
\rho(E)= & c_{N} \sqrt{1-\left(E / E_{0}\right)^{2}} \prod_{k=1}^{\infty}\left[1-4 \frac{E^{2}}{E_{0}^{2}} \frac{1}{2+|\eta|^{2 k}+|\eta|^{-2 k}}\right] \\
& \times\left[1-4 \frac{E^{2}}{E_{0}^{2}} \frac{1}{2-|\eta|^{2 k-1}-|\eta|^{-2 k+1}}\right] \\
= & c_{N} \exp \left[\frac{1}{2} \sum_{k=-\infty}^{\infty} \log \left[1-4 \frac{E^{2}}{E_{0}^{2}} \frac{1}{2+|\eta|^{2 k}+|\eta|^{-2 k}}\right]\right. \\
& \left.+\log \left[1-4 \frac{E^{2}}{E_{0}^{2}} \frac{1}{2-|\eta|^{2 k-1}-|\eta|^{-2 k+1}}\right]\right] . \quad \text { (A1) }
\end{aligned}
$$

With the normalization constant $c_{N}$ given by

$$
c_{N}=\frac{2^{N / 2}}{\pi \sigma}(1+\eta) \sqrt{1-\eta} \prod_{k=1}^{\infty} \frac{1-\eta^{2 k+2}}{1-\eta^{2 k+1}}
$$

the spectral density is normalized to $2^{N / 2}$. After a Poisson resummation, the expression can be rewritten as

$$
\begin{aligned}
\rho(E)= & c_{N} \exp \left[\frac{1}{2} \sum_{n=-\infty}^{\infty} \int d x e^{2 \pi i n x} \log \left[1-\frac{E^{2}}{E_{0}^{2}} \frac{1}{\cosh ^{2} x \log |\eta|}\right]\right. \\
& \left.+\log \left[1+\frac{E^{2}}{E_{0}^{2}} \frac{1}{\sinh ^{2}\left(x-\frac{1}{2}\right) \log |\eta|}\right]\right] .
\end{aligned}
$$

Both integrals can be evaluated analytically:

$$
\begin{aligned}
\frac{1}{2} \int d x e^{2 \pi i n x} \log \left[1+\frac{E^{2}}{E_{0}^{2}} \frac{1}{\sinh ^{2}\left(x-\frac{1}{2}\right) \log |\eta|}\right] \\
=\frac{(-1)^{n}}{2|n|}\left(1-\exp \left[\frac{2 \pi|n|}{\log |\eta|}\left|\arcsin \left(\frac{E}{E_{0}}\right)\right|\right]\right),
\end{aligned}
$$

and

$$
\begin{gathered}
\frac{1}{2} \int d x e^{2 \pi i n x} \log \left[1-\frac{E^{2}}{E_{0}^{2}} \frac{1}{\cosh ^{2} x \log |\eta|}\right] \\
=-\frac{1}{2} \frac{1-\cosh \left[\frac{2 \pi|n|}{\log |\eta|} \arcsin \left(E / E_{0}\right)\right]}{n \sinh \left(n \pi^{2} / \log |\eta|\right)} .
\end{gathered}
$$

The case $n=0$ has to be considered separately. After shifting $x$ by $1 / 2$ we obtain

$$
\frac{1}{2} \int d x \log \left[1+\frac{E^{2}}{E_{0}^{2}} \frac{1}{\sinh ^{2} x \log |\eta|}\right] .
$$

Using $x|\log \eta|$ as the new integration variable, this can be written as

$$
\begin{aligned}
- & \frac{1}{2} \frac{1}{\log |\eta|} \int d x \log \left[1+\frac{E^{2}}{E_{0}^{2}} \frac{1}{\sinh ^{2} x}\right] \\
= & +\frac{1}{8 \log |\eta|}\left(\log \left(e^{-2 i \phi}\right)\left(-2 \pi i \operatorname{sign}(\phi)+\log e^{2 i \phi}\right)\right. \\
& \left.+\log \left(e^{2 i \phi}\right)\left(2 \pi i \operatorname{sign}(\phi)+\log e^{-2 i \phi}\right)\right) \\
= & -\frac{\pi\left|\arcsin \left(E / E_{0}\right)\right|}{\log |\eta|}+\frac{\arcsin ^{2}\left(E / E_{0}\right)}{\log |\eta|},
\end{aligned}
$$

where $\phi=\arcsin \left(E / E_{0}\right)$. Ignoring exponentially small contributions, for $\left|E / E_{0}\right|<1$ the terms with $n \neq 0$ in Eq. (A4) can be summed into

$$
\log \left(\left(1+e^{2 \pi\left|\arcsin \left(E / E_{0}\right)\right| / \log |\eta|}\right) / 2\right) .
$$

For large $N$ away from the edge of the spectrum the leading contribution to the spectral density is dominated by the $n=0$ term resulting in the asymptotic form for the spectral density,

$$
\begin{aligned}
\rho_{\text {asym }}(E)= & c_{N} \cosh \left(\frac{\pi \arcsin \left(E / E_{0}\right)}{\log |\eta|}\right) \\
& \times \exp \left[2 \frac{\arcsin ^{2}\left(E / E_{0}\right)}{\log |\eta|}\right] .
\end{aligned}
$$

The large $N$ limit of the normalization constant is determined by

$$
\int d E \rho_{\text {asym }}(E) d E=2^{N / 2} .
$$

For large $N$ the integral can be evaluated by a saddle point approximation. Using that $\log |\eta| \sim-2 q^{2} / N$ in this limit we find

$$
c_{N}=e^{N / 2 \log 2-N \pi^{2} / 16 q^{2}},
$$

which gives exactly the leading order $1 / q^{2}$ correction to the zero temperature entropy [52]. 


\section{APPENDIX B: THE Q-HERMITE APPROXIMATION FOR THE RESOLVENT OF THE SYK MODEL}

The resolvent of a Hamiltonian $H$ is defined by

$$
G(z)=\frac{1}{\mathcal{N}} \operatorname{Tr} \frac{1}{z+H},
$$

where $\mathcal{N}$ is the dimension of the Hilbert space. If $\left|z / \lambda_{k}\right|>1$ for all eigenvalues $\lambda_{k}$ of $H$ it can be expressed in terms of the moments of $H$. For a Hamiltonian for which the odd moments vanish, such as the SYK model, we obtain

$$
G(z)=\sum_{p=0}^{\infty} \frac{M_{2 p}}{z^{2 p+1}} .
$$

This sum has a finite radius of convergence due to the fact that it has a cut along the support of the spectrum (for a Gaussian the radius of convergence is zero). However, it may be possible to analytically continue $G(z)$ inside the radius of convergence. This can be done using the resummation

$\frac{1}{z^{2 k+1}}=\sum_{j=0}^{\infty}(-1)^{j}\left(\begin{array}{c}2 k+j \\ j\end{array}\right) \frac{1}{\left(z / 2+\sqrt{z^{2} / 4-1}\right)^{2(j+k)+1}}$.

The sum is convergent on the imaginary axis. To prove this identity we start from the binomial expansion

$$
\frac{1}{\left(e^{x}+e^{-x}\right)^{2 k+1}}=\sum_{j=0}^{\infty}\left(\begin{array}{c}
2 k+j \\
j
\end{array}\right) e^{-x(2 j+2 k+1)} .
$$

Defining $z$ as

$$
z=e^{x}+e^{-x}
$$

we can solve $e^{x}$ by

$$
e^{x}=\frac{z}{2} \pm \sqrt{z^{2} / 4-1}
$$

resulting in

$$
\frac{1}{z^{2 k+1}}=\sum_{j=0}^{\infty}(-1)^{j}\left(\begin{array}{c}
2 k+j \\
j
\end{array}\right) \frac{1}{\left(z / 2 \pm \sqrt{z^{2} / 4-1}\right)^{2(j+k)+1}} .
$$

For large $z$ only the positive sign on the right-hand side reproduces the $1 / z^{2 k+1}$ term so that the correct analytical continuation is given by Eq. (B3).
Because the spectrum of the SYK Hamiltonian is symmetric under $\lambda_{k} \rightarrow-\lambda_{k}$, the resolvent on the imaginary axis is purely imaginary and

$$
i G(i s)=\frac{1}{\mathcal{N}} \sum_{\lambda_{k}>0} \frac{2 s}{\lambda_{k}^{2}+s^{2}} .
$$

Using the analytical continuation (B3) we obtain

$$
\begin{aligned}
i G(i s)= & \sum_{p=0}^{\infty}(-1)^{p} \frac{M_{2 p}}{M_{2}^{p}} \frac{1}{s^{2 p+1}} \\
= & \sum_{p=0}^{\infty} \frac{M_{2 p}}{M_{2}^{p}} \sum_{j=0}^{\infty}(-1)^{p}\left(\begin{array}{c}
2 p+j \\
j
\end{array}\right) \\
& \times \frac{1}{\left(s / 2+\sqrt{s^{2} / 4+1}\right)^{2(j+p)+1}} .
\end{aligned}
$$

The second line of (B9) also converges when $s$ is small. In the Q-Hermite case the moments are given by

$$
\frac{M_{2 p}}{M_{2}^{p}}=\frac{1}{(1-\eta)^{p}} \sum_{k=-p}^{p}(-1)^{k} \eta^{k(k-1) / 2}\left(\begin{array}{c}
2 p \\
p+k
\end{array}\right) .
$$

We will show next that the result (B9) can be simplified to

$i G(i s)=\sqrt{1-\eta} \sum_{k=0}^{\infty} \frac{\eta^{k(k+1) / 2}}{\left(\frac{s \sqrt{1-\eta}}{2}+{\sqrt{\frac{s^{2}(1-\eta)}{4}+1}}^{2 k+1}\right.}$.

To prove this, we first shift $s \rightarrow s \sqrt{1-\eta}$ which cancels against the prefactor of the Q-Hermite moments as can be seen from the first equation of Eq. (B9). Then we use the identity (see [64])

$\frac{1}{\left(\frac{s}{2}+\sqrt{\frac{s^{2}}{4}+1}\right)^{2 k+1}}=\sum_{j=0}^{\infty} \frac{2 k+1}{2 k+2 j+1}\left(\begin{array}{c}2 j+2 k+1 \\ j\end{array}\right) \frac{1}{s^{2 k+2 j+1}}$

to rewrite (B9) as a double sum which can be rewritten as a sum over $p \equiv k+j$ fixed and a sum over $j$ which runs from 0 to $p$. By splitting the sum over $k$ from $-p$ to $p$ in the expression for the Q-Hermite moments into a sum from 0 to $p$ and a sum form $-p$ to -1 we see that the two expressions for the resolvent are the same.

A scale can be reintroduced by the substitution

$$
i G(i s) \rightarrow \frac{1}{\sigma} i G(i s / \sigma) \text {. }
$$


[1] E. Wigner, Math. Proc. Cambridge Philos. Soc. 47, 790 (1951).

[2] F. Dyson, J. Math. Phys. (N.Y.) 3, 140 (1962).

[3] F. Dyson, J. Math. Phys. (N.Y.) 3, 157 (1962).

[4] F. Dyson, J. Math. Phys. (N.Y.) 3, 166 (1962).

[5] F. Dyson, J. Math. Phys. (N.Y.) 3, 1191 (1962).

[6] F. Dyson, J. Math. Phys. (N.Y.) 13, 90 (1972).

[7] O. Bohigas, M. J. Giannoni, and C. Schmit, Phys. Rev. Lett. 52, 1 (1984).

[8] E. V. Shuryak and J. J. M. Verbaarschot, Nucl. Phys. A560, 306 (1993).

[9] J. J. M. Verbaarschot and I. Zahed, Phys. Rev. Lett. 70, 3852 (1993).

[10] J. J. M. Verbaarschot, Phys. Rev. Lett. 72, 2531 (1994).

[11] T. Banks and A. Casher, Nucl. Phys. B169, 103 (1980).

[12] B. L. Altshuler and B. I. Shklovskii, Zh. Eksp. Teor. Fiz. 91, 220 (1986) [ Sov. Phys. JETP 64, 127 (1986).]

[13] B. L. Altshuler, Y. Gefen, A. Kamenev, and L. S. Levitov, Phys. Rev. Lett. 78, 2803 (1997).

[14] J. C. Osborn and J. J. M. Verbaarschot, Phys. Rev. Lett. 81, 268 (1998).

[15] J. French and S. Wong, Phys. Lett. 33B, 449 (1970).

[16] J. French and S. Wong, Phys. Lett. 35B, 5 (1971).

[17] O. Bohigas and J. Flores, Phys. Lett. 34B, 261 (1971).

[18] O. Bohigas and J. Flores, Phys. Lett. 35B, 383 (1971).

[19] V. K. B. Kota, Embedded Random Matrix Ensembles in Quantum Physics (Springer, New York, 2014), vol. 884.

[20] A. Kitaev, A simple model of quantum holography, KITP strings seminar and Entanglement 2015 program, 12 February, 7 April and 27 May 2015, http://online.kitp.ucsb .edu/online/entangled15/.

[21] S. Sachdev, Phys. Rev. Lett. 105, 151602 (2010).

[22] S. Sachdev and J. Ye, Phys. Rev. Lett. 70, 3339 (1993).

[23] J. Maldacena, S. H. Shenker, and D. Stanford, J. High Energy Phys. 08 (2016) 106.

[24] J. Maldacena and D. Stanford, Phys. Rev. D 94, 106002 (2016).

[25] K. Jensen, Phys. Rev. Lett. 117, 111601 (2016).

[26] J. Polchinski and V. Rosenhaus, J. High Energy Phys. 04 (2016) 001.

[27] J. Maldacena, D. Stanford, and Z. Yang, Prog. Theor. Exp. Phys. 2016, 12C104 (2016).

[28] O. Parcollet, A. Georges, G. Kotliar, and A. Sengupta, Phys. Rev. B 58, 3794 (1998).

[29] A. Georges, O. Parcollet, and S. Sachdev, Phys. Rev. B 63, 134406 (2001).

[30] A. Jevicki, K. Suzuki, and J. Yoon, J. High Energy Phys. 07 (2016) 007.

[31] A. Jevicki and K. Suzuki, J. High Energy Phys. 11 (2016) 046.

[32] A. M. Garcia-Garcia and J. J. M. Verbaarschot, Phys. Rev. D 96, 066012 (2017).

[33] J. S. Cotler, G. Gur-Ari, M. Hanada, J. Polchinski, P. Saad, S. H. Shenker, D. Stanford, A. Streicher, and M. Tezuka, J. High Energy Phys. 05 (2017) 118.

[34] D. Bagrets, A. Altland, and A. Kamenev, Nucl. Phys. B911, 191 (2016).

[35] D. Bagrets, A. Altland, and A. Kamenev, Nucl. Phys. B921, 727 (2017).
[36] A. M. Garcia-Garcia and J. J. M. Verbaarschot, Phys. Rev. D 94, 126010 (2016).

[37] C. Krishnan, K. V. P. Kumar, and S. Sanyal, J. High Energy Phys. 06 (2017) 036.

[38] J. Maldacena, D. Stanford, and Z. Yang, Prog. Theor. Exp. Phys. (2016) 12C104.

[39] A. Almheiri and J. Polchinski, J. High Energy Phys. 11 (2015) 1

[40] C. Teitelboim, Phys. Lett. 126B, 41 (1983).

[41] R. Jackiw, Nucl. Phys. B252, 343 (1985).

[42] D. J. Gross and V. Rosenhaus, J. High Energy Phys. 02 (2017) 093.

[43] C. Krishnan, K. V. P. Kumar, and D. Rosa, J. High Energy Phys. 01 (2018) 064.

[44] R. A. Davison, W. Fu, A. Georges, Y. Gu, K. Jensen, and S. Sachdev, Phys. Rev. B 95, 155131 (2017).

[45] S.-K. Jian and H. Yao, Phys. Rev. Lett. 119, 206602 (2017).

[46] G. Turiaci and H. Verlinde, J. High Energy Phys. 10 (2017) 167.

[47] S. R. Das, A. Jevicki, and K. Suzuki, J. High Energy Phys. 09 (2017) 017.

[48] S. R. Das, A. Ghosh, A. Jevicki, and K. Suzuki, arXiv: 1712.02725 .

[49] A. M. Garcia-Garcia, B. Loureiro, A. Romero-Bermúdez, and M. Tezuka, arXiv:1707.02197.

[50] X.-Y. Song, C.-M. Jian, and L. Balents, Phys. Rev. Lett. 119, 216601 (2017).

[51] X. Chen, R. Fan, Y. Chen, H. Zhai, and P. Zhang, Phys. Rev. Lett. 119, 207603 (2017).

[52] W. Fu, D. Gaiotto, J. Maldacena, and S. Sachdev, Phys. Rev. D 95, 026009 (2017); 95, 069904 (2017).

[53] C. Peng, M. Spradlin, and A. Volovich, J. High Energy Phys. 05 (2017) 062.

[54] C. Peng, M. Spradlin, and A. Volovich, J. High Energy Phys. 10 (2017) 202.

[55] T. Li, J. Liu, Y. Xin, and Y. Zhou, J. High Energy Phys. 06 (2017) 111.

[56] T. Kanazawa and T. Wettig, J. High Energy Phys. 09 (2017) 050 .

[57] N. Hunter-Jones and J. Liu, arXiv:1710.08184.

[58] P. Narayan and J. Yoon, arXiv:1712.02647.

[59] J. Murugan, D. Stanford, and E. Witten, J. High Energy Phys. 08 (2017) 146.

[60] E. Witten, arXiv:1610.09758.

[61] I. R. Klebanov and G. Tarnopolsky, Phys. Rev. D 95, 046004 (2017).

[62] A. Altland and M. R. Zirnbauer, Phys. Rev. B 55, 1142 (1997).

[63] S. Förste and I. Golla, Phys. Lett. B 771, 157 (2017).

[64] M. E. Ismail, D. Stanton, and G. Viennot, Eur. J. Combinatorics 8, 379 (1987).

[65] L. Erdős and D. Schröder, Math. Phys. Anal. Geom. 17, 9164 (2014).

[66] T. Nagao and P. J. Forrester, Nucl. Phys. B435, 401 (1995).

[67] D. A. Ivanov, J. Math. Phys. (N.Y.) 43, 126 (2002).

[68] A. Altland and D. Bagrets, Nucl. Phys. B930, 45 (2018).

[69] Y. Alhassid and R. D. Levine, Phys. Rev. A 46, 4650 (1992). 
[70] F. Borgonovi, F. M. Izrailev, L. F. Santos, and V. G. Zelevinsky, Phys. Rep. 626, 1 (2016).

[71] M. Távora, E. J. Torres-Herrera, and L. F. Santos, Phys. Rev. A 95, 013604 (2017).
[72] E. J. Torres-Herrera, A. M. García-García, and L. F. Santos, Phys. Rev. B 97, 060303 (2018).

[73] N. Argaman, Y. Imry, and U. Smilansky, Phys. Rev. B 47, 4440 (1993). 